\title{
SAR Image Superresolution via 2-D Adaptive Extrapolation ${ }^{\text {is }}$
}

\author{
ALEJANDRO E. BRITO \\ abrito@dsp.ece.utep.edu \\ Department of Electrical and Computer Engineering, The University of Texas at El Paso, El Paso, TX 79968 \\ USA \\ SHIU H. CHAN \\ Department of Electrical and Computer Engineering, The University of Texas at El Paso, El Paso, TX 79968 \\ USA \\ SERGIO D. CABRERA \\ cabrera@ece.utep.edu \\ Department of Electrical and Computer Engineering, The University of Texas at El Paso, El Paso, TX 79968 \\ USA
}

Received December 1, 2000; Revised March 27, 2002; Accepted April 9, 2002

Abstract. In this paper, we present a description of a nonparametric two dimensional (2-D) procedure to extrapolate a signal, an extension of the Adaptive Weighted Norm Extrapolation (AWNE) method, and illustrate its application to SAR image formation. The benefits of the AWNE procedure are shown for synthetic data and for MSTAR data. Once the phase history is recovered, the AWNE method is applied to a subaperture or to the full set of frequency samples to extrapolate them to a larger aperture from which a superresolved complex SAR image is obtained. Use of the 2-D AWNE procedure proves to be superior to its one-dimensional separable version by reducing undesirable effects such as sidelobe interference, and variability in energy of the extrapolated data from row to row and column to column. To assess the performance of AWNE in enhancing prominent scatterers, reducing speckle, and suppressing clutter, we compare the superresolved images to the images formed with the traditional Fourier technique starting from the same phase history data. Fourier images are also compared with superresolved images formed using less data in order to assess the quality of the extrapolation and to quantify the method's ability to recover lost resolution. We illustrate performance by visual comparison and by the use of a geometric constellation of prominent point scatterers of the targets extracted from the images. A brief comparison with the 2-D versions of Capon and the linear prediction methods is illustrated and a hybrid AWNE/Capon approach is proposed.

Key Words: image formation, superresolution, MSTAR, synthetic aperture radar, extrapolation

\section{Introduction}

There is an increasing interest in the use of superresolution methods to form Synthetic Aperture Radar (SAR) images [1], [2], [3], [4], [5], [6], [7], [8]. The motivation for applying these methods to SAR, as discussed by DeGraaf [1], is to improve resolution,

\footnotetext{
This work was supported by ARO Grants DAAH04-95-1-0494 and DAAD19-99-1-0012, and by a grant
} from the Texas Instruments Foundation. 
to suppress sidelobe artifacts, and to reduce speckle in comparison with traditional Fourier imaging techniques. Improvements in resolution of prominent scatterers, speckle reduction, enhancement of contrast, and CFAR detectability of targets in clutter are among the reported results [1]. Superresolution methods have also proven to increase significantly the performance of Automatic Target Recognition (ATR) systems [2]. A one-dimensional (1-D) nonparametric procedure to extrapolate a narrowband signal, denoted Adaptive Weighted Norm Extrapolation (AWNE) [9], has been used to enhance resolution in SAR imaging by row-column processing [7]. In [8], we reported the use of a two-dimensional (2-D) version of the algorithm where the emphasis was on objectively quantifying the quality of the superresolved images using a CFAR detector to segment target and clutter in order to compute metrics, such as peak location and matching, when comparing to a reference image. The AWNE procedure is based on the iterative use of re-weighting in minimum weighted norm extrapolation to produce a spectrally concentrated stationary extension of the given data [9]. Recently, a similar procedure has been extensively analyzed and promoted for use in geophysical data processing problems [10]. It has also been recently re-cast and related to a general procedure to solve a linear inverse problem with sparseness constraints [11].

In this paper, we present a description of the two-dimensional (2-D) version of AWNE and propose its application in SAR imaging using data obtained from the MSTAR [12] targets database of images. Once the phase history is obtained by reversing the windowing and Discrete Fourier Transform (DFT) steps of the Fourier image formation, the AWNE method is applied to a subaperture or to the full set of frequency samples to extrapolate them to a larger aperture. Then, the Inverse DFT is applied to obtain the new complex SAR image. The use of the 2-D AWNE procedure minimizes potential artifacts that can be introduced when the 1-D version [7] is used.

To experimentally assess the performance of the 1-D and 2-D AWNE in enhancing prominent scatterers, reducing speckle, and suppressing clutter, we compare the superresolved (SR) images to images formed with the traditional Fourier techniques using the same amount of data. We also evaluate images formed with less data to assess the quality of the extrapolation and to quantify the benefits of using superresolution if less than the desired amount of phase history data is available. Thus, in our first set of experiments, we use a subaperture of the frequency samples to evaluate the performance of the 2-D AWNE in this handicapped situation in comparison to the 1-D AWNE algorithm and to the traditional Fourier approach. The other focus of our experiments is the illustration of the potential advantages of going beyond the traditional limits of resolution by extrapolating the full aperture of phase history to a larger size. We evaluate the results by visual comparison and the geometric constellation of prominent point scatterers of the targets extracted from the images. Finally, an experimental comparison of superresolved images generated by the 2-D AWNE, the Capon's minimum variance, and the 2-D linear prediction (AR) methods is presented. Particular features of the SR images are discussed and a hybrid scheme combining the 2-D AWNE and MVM methods is proposed to take advantage of the best features of each method. 


\section{The Adaptive Extrapolation Method}

\subsection{Minimum Weighted Norm Extrapolation}

\subsubsection{Previous Related Work}

The nonparametric extrapolation problem has been the subject of extensive research and a diverse array of iterative and noniterative methods have been devised [9], [13], [14], [15], [16], [17], [18], [19]. Recently, generalizations of some of these methods to multidimensional signals with discontiguous passbands and discontiguous timeconcentration regions have been developed [19]. Since the nonparametric extrapolation problem always admits an infinite number of solutions due to its underdetermined nature, several schemes to choose a solution have been proposed and good reviews of the most popular methodologies for this selection can be found in [17], [18]. Of particular interest are the minimum norm (energy) extrapolation algorithms [9], [13], [14], [15], [16] where the signal with the least energy is chosen from the set of admissible extrapolations. Typically, conventional least squares stabilization theory for dealing with ill-conditioned problems is used. Also, some type of constraint or assumed a priori information is used in order to reach a desirable type of solution. Frequency weighting based extrapolation algorithms [9], [15], [16], [17] are particular cases devised in order to obtain a minimum weighted norm (energy) solution that matches the given data (data consistent) and reflects the available frequency profile information, which describes the desirable energy distribution. The frequency weighting can be a given profile [15], [16] or it can be directly inferred from the data [9] via a spectrum estimator. These algorithms can be considered more general than the traditional band-limited extrapolation algorithms [13], [14] in the sense that ideal spectral support information is not necessary. However, the main data consistency constraint is always present in both types of algorithms.

\subsubsection{Mathematical Formulation}

We denote a general set of $L$ given discrete-time data samples as $\left\{x\left(m_{1}\right), x\left(m_{2}\right), \ldots, x\left(m_{L}\right)\right\}$, where all $m_{k}$ values are integers. A frequency weighting function $Q(f)$ can be used to define an inner product in the linear space $V$ of signals with spectral support $B$, where $B \subset[-0.5,0.5]$. Assuming that $Q(f)>0$ for $f \in B$, and that $Q(f)=0$ otherwise, this inner product is

$$
\langle x(n), y(n)\rangle_{Q}=\int_{f \in B} \frac{X(f) Y^{*}(f)}{Q(f)} d f .
$$

The infinite set $D$ of valid extrapolations is the subset of signals in $V$ which go through the given time samples. An optimal unique choice $\hat{x}(n)$ for the associated linearly constrained optimization problem [11] is the Minimum Weighted Norm Extrapolation 
(MWNE), which solves

$$
\min _{x(n) \in D}\left\{\int_{f \in B} \frac{|X(f)|^{2}}{Q(f)} d f\right\}=\|\hat{x}(n)\|_{Q}^{2}=\int_{f \in B} \frac{|\hat{X}(f)|^{2}}{Q(f)} d f .
$$

The extrapolation $\hat{x}(n)$ is thus the result of an optimal spectral shape match of $|\hat{X}(f)|^{2}$ to $Q(f)$. That is, $Q(f)$ can be used as a tool to influence the choice of $\hat{x}(n)$ from $D$ and it provides a means for obtaining high resolution. The weighting function $Q(f)$ may not be available as a priori information but may be a spectrum estimate obtained using a simple technique that only requires the available time samples at hand, such as the periodogram. It can be shown [9] that defining the autocorrelation sequence $q(n)$ as the inverse-Fourier transform of $Q(f)$, a closed form solution for $\hat{x}(n)$ exists. This is the MWNE extrapolation which is a linear expansion of the form

$$
\widehat{x}(n)=\sum_{i=1}^{L} b_{i} q\left(n-m_{i}\right)
$$

where the $b_{i}$ 's are the extrapolation coefficients that can be obtained directly from the $L$-constraints that make $\hat{x}(n)$ a data consistent extrapolation. To solve for the $b_{i}$ 's, a $L \times L$ Gram matrix $G$ is defined having entries $(G)_{r, c}=q\left(m_{r}-m_{c}\right)$ for row $r$ and column $c$. In the case of uniformly-spaced, contiguous samples, $G$ is a Hermitian Toeplitz matrix since $(G)_{r, c}=q(r-c)$. From Eq. (1), we note that the resulting extrapolation $\hat{x}(n)$ has a length given by $L+N_{q}-1$, for an autocorrelation $q(n)$ of length $N_{q}=2 J-1$ and a given parameter $J \geq L$ that controls the desired extrapolation length. From Eq. (1), the frequency domain form of MWNE is given by

$$
\hat{X}(f)=Q(f) \sum_{i=1}^{L} b_{i} e^{-j 2 \pi f m_{i}} .
$$

The frequency weighting $Q(f)$ summarizes the prior or inferred knowledge and is a smooth function if $q(n)$ has finite duration as we assume. This spectrum heavily influences the solution, see Eq. (2).

\subsection{Adaptive Weighted Norm Extrapolation (AWNE)}

In general, the minimum norm extrapolation solution concentrates the energy around the observation indices, suppressing energy outside this region [19]. A good extrapolation results from this method only if most of the energy of the signal to reconstruct (extrapolate/ interpolate) is actually concentrated in the region of the observations. This assumption is not always appropriate, specially in the recovery of sinusoidal signals. Therefore, schemes can be devised to improve the quality of the reconstructed signal. One algorithm that incorporates the benefits of the minimum energy solution and tries to overcome the concentration of energy around the observation indices by generating longer extrapolation sequences through an iterative procedure is the AWNE method [9]. AWNE, in each 


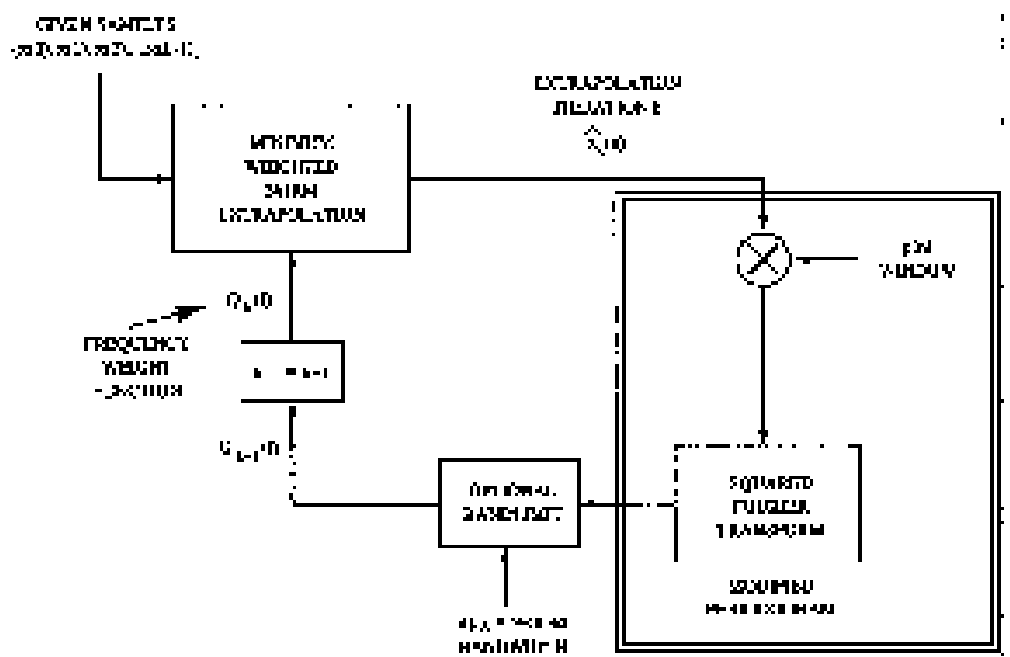

Figure 1. 1-D AWNE block diagram.

iteration, finds a minimum weighted norm extrapolation solution and uses it to compute a new, more concentrated weighting function. The fundamental idea is to infer the frequency weighting information from the extrapolation solution of the previous iteration in order to refine the current spectrum profile by making it more concentrated. Consequently, this increases the energy in the overall time (space) domain. This method provides a better solution for sinusoidal signals [11] than any of the two-step minimum energy extrapolation methods [13], [14], [15], [16].

AWNE is an iterative process that uses the original data, as well as some of the newly computed (extrapolated) data samples, to obtain a new $Q(f)$ at each iteration for use in the next one. To reduce sidelobe effects and have control of the resultant extrapolation length, the spectrum in the $(k+1)$-th estimate is computed via the modified periodogram as $Q_{k+1}(f)=\left|\mathcal{F}\left\{\hat{x}_{k}(n) \cdot p(n)\right\}\right|^{2}$, where $p(n)$ can be any standard non-negative symmetric window. There is a direct relationship between the resolution that the extrapolation can achieve and the type of window $p(n)$ used as well as a trade off between the stability of the extrapolation and the sidelobe attenuation implied by the window type. Analysis of these issues has been reported in [11], [20], [21] where the extrapolation resolution is related to an imposed window which is characterized as a Tukey-like window and a new algorithm has been devised to undo this effect achieving the highest resolution possible [11], [22].

The AWNE procedure is illustrated in Figure 1 for the case of contiguous data samples in the range $0 \leq n \leq L-1$, indicating that a priori spectral support (bandwidth) information can also be incorporated, if available. Using a fixed window $p(n)$ of length $J \geq L$, the iterative procedure produces increasing resolution until little change occurs between one iteration and the next. The final result of the AWNE procedure is the extrapolated signal at the last iteration. The maximum possible length $N_{E}$ of this signal, and that of all intermediate results, is determined by the window length parameter $J$, 

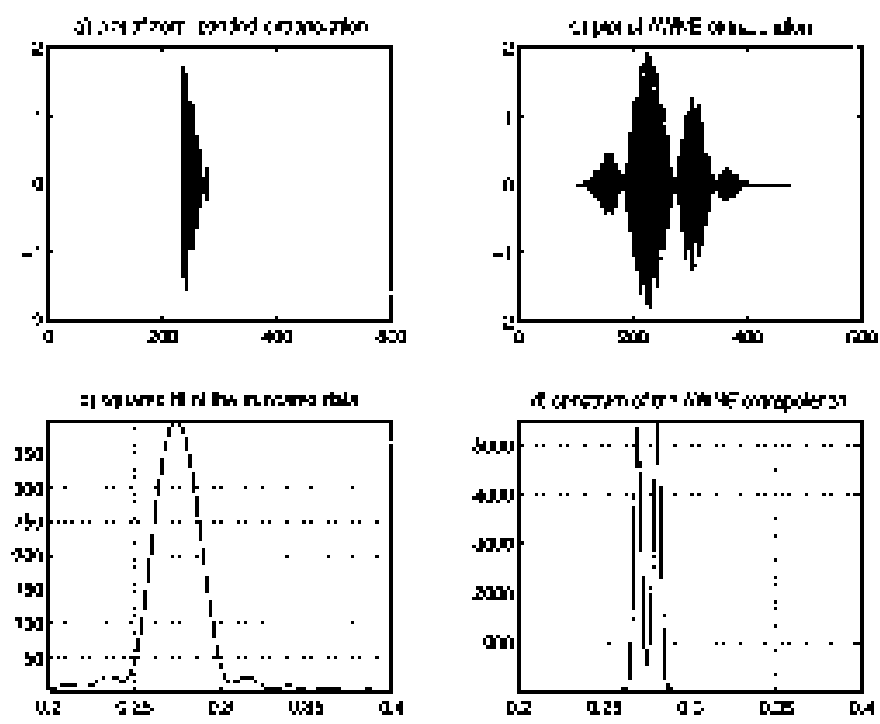

Figure 2. 1-D extrapolation example.

which produces an autocorrelation $q(n)$ of length $N_{q}=2 J-1$. Since the number of given data samples is $L$, this leads to (see Eq.(1))

$$
N_{E}=N_{q}+L-1=2 J+L-2 .
$$

To save computations, the process can also be performed for a fixed number of iterations; typically 3-5 iterations, stopping before the convergence is reached. Such an approach has been used in some previous experiments with SAR imaging [7]. Because the length of all signals involved is known and finite, a DFT of length $N_{D}$ can be used, with $N_{D} \geq N_{E}$. The zero-th extrapolation resulting from $Q_{0}(f)=1$ is the zero-padded data $\hat{x}_{0}(n)$. The first iteration (non-trivial) of the procedure uses a weight $Q_{1}(f)$ which is the modified periodogram computed using only the zero-padded given data. As a consequence, the first extrapolation $\hat{x}_{1}(n)$ will be a linear combination of shifted versions of the autocorrelation $q_{1}(n)$ of the windowed zero-padded data. This result is a significantly different extrapolation than the zero-padded data. The length of $\hat{x}_{1}(n)$ is $N_{1}=3 L-2$ which corresponds to $N_{E}$ when $J=L$ in Eq. (3). Although we are concentrating on contiguous uniformly spaced data samples, it is important to mention that AWNE can be applied to interpolate missing gaps of samples [9]. Figure 2 shows the results of applying the AWNE method to extrapolate 45 samples of the signal

$$
x(n)=e^{j 2 \pi(0.27 n)}+e^{j 2 \pi\left(0.28 n+\frac{\pi}{4}\right)} .
$$

In Figure 2, the real part of the 45 samples of the original signal are shown in a); the result of the AWNE method is shown in b); the spectrum of the truncated data is 
shown in c); and the spectrum of the extrapolated data is shown in d). This example shows the frequency domain high resolution capability of AWNE.

\subsection{Extension to a Two-Dimensional Version}

\subsubsection{Motivation}

In this paper, we provide an extension to 2-D of the 1-D AWNE method focusing on the case of uniformly spaced samples and on its use for SAR superresolution. We recently reported [7] the use of 1-D AWNE to enhance the resolution of MSTAR SAR images for further processing. We performed experiments to evaluate the performance of AWNE on SAR images taken from the MSTAR database focusing on the effects of using a reduced set of phase history samples to attempt to re-generate the same images, under this handicapped situation, with the help of AWNE. We used several criteria [7] to compare the superresolved images to the original MSTAR images and showed how the method behaves as the phase history subaperture block size changes. Despite the handicapped situation considered, we obtained SAR images of higher resolution with enhanced prominent scatterers and reduced speckle. We also found that the same statistical model (Weibull) can be used in the CFAR detector of the target region on both the original and the SR image. In this paper, we derived and use a 2-D version of AWNE to reduce the peak location mismatch, attenuate better the sidelobes, and produce overall better images because the local 2-D dependencies are taken into account. In addition, the 2-D AWNE version can be compared more fairly to other 2-D superresolution methods [1], [3], [4], [5], [6] used for SAR image formation.

\subsubsection{Two-Dimensional AWNE}

Following the discussion of the previous sections we can see that the extension of AWNE to a 2-D version for discrete-time signals can be done starting from the minimum weighted norm extrapolation procedure so that Eq. (1) now takes the form

$$
\widehat{x}(n, m)=\sum_{i=1}^{L} \sum_{j=1}^{L} b_{i, j} q\left(n-n_{i}, m-m_{j}\right),
$$

where we now have $L \times L$ extrapolation coefficients, the $b_{i, j}$ 's, matching the $L \times L$ number of given data samples of $x(n, m)$, which are the constraints that make $\hat{x}(n, m)$ a valid extrapolation. For contiguous uniformly spaced data samples, a vector $y$ containing the given data samples and a matrix $G$ are used to solve for the vector $\underline{b}$ of $b_{i, j}{ }^{\bar{\prime}}$ 's in Eq. (4). The Gram matrix $G$ has $L \times L$ blocks denoted $g(k)$ so that

$$
G=\left[\begin{array}{cccc}
g(0) & g(-1) & \cdots & g(-L+1) \\
g(1) & g(0) & \cdots & g(-L+2) \\
\vdots & \vdots & \vdots & \vdots \\
g(L-1) & g(L-2) & \cdots & g(0)
\end{array}\right],
$$


and each block $g(k)$ is also of dimension $L \times L$, having the form

$$
g(k)=\left[\begin{array}{cccc}
q(0, k) & q(-1, k) & \cdots & q(-L+1, k) \\
q(1, k) & q(0, k) & \cdots & q(-L+2, k) \\
\vdots & \vdots & \vdots & \vdots \\
q(L-1, k) & q(L-2, k) & \cdots & q(0, k)
\end{array}\right] .
$$

Clearly, the block matrix $G$ is Hermitian and has a block-Toeplitz structure since $q(n, m)=q^{*}(-n,-m)$. However, the blocks are not in general Hermitian. Regarding the iterative part of the process, the window $p(n)$ used in the 1-D AWNE version has various extensions to 2-D including the circularly symmetric 2-D window [23]. The rest of the components defined in Figure 1 can be extended in a simple way because they all have 2-D counterparts.

We can summarize next all the computations of the algorithm whose given items are the $L \times L$ samples of $x(n, m)$, the window diameter (length) $J \geq L$ centered on the data, a maximum number of iterations $K_{0}$ or a stopping tolerance $\varepsilon$ :

Step 0 . Form the 2-D circularly symmetric window $p(n, m)$ of diameter $J$ and zero-pad it to size $N_{E} \times N_{E}$ where $N_{E}=2 J+L-2$.

Step 1. Extend $x(n, m)$ to a zero-padded version $x_{z}(n, m)$ of size $N_{E} \times N_{E}$ preserving the original samples in the center of $x_{z}(n, m)$.

Step 2. The initial extrapolation for $k=0$ is given by $\hat{x}_{0}(n, m)=x_{z}(n, m)$.

Step 3. Increment $k$ and replace the previous extrapolation with a windowed version

$$
\widehat{x}_{k-1}(n, m)=\widehat{x}_{k-1}(n, m) \cdot p(n, m) .
$$

Step 4. Obtain the 2-D autocorrelation

$$
q_{k}(n, m)=\hat{x}_{k-1}(n, m) * \hat{x}_{k-1}^{*}(-n,-m) .
$$

Step 5. Form the block-Toeplitz matrix $G$ according its definition in Eqs. (5) and (6). Step 6. Solve for $\underline{b}$ from

$$
G \cdot \underline{b}=\underline{y}
$$

where $\underline{b}$ is the vector of ordered extrapolation coefficients and $y$ is a similarly ordered set of data samples.

Step 7. Compute the new extrapolation as

$$
\hat{x}_{k}(n, m)=\sum_{i=1}^{L} \sum_{j=1}^{L} b_{i, j} q_{k}(n-i, m-j) .
$$

Step 8. Stop if $\left\|\widehat{x}_{k}(n, m)-\widehat{x}_{k-1}(n, m)\right\|<\varepsilon$ or $k=K_{0}$, otherwise go to Step 3 . 

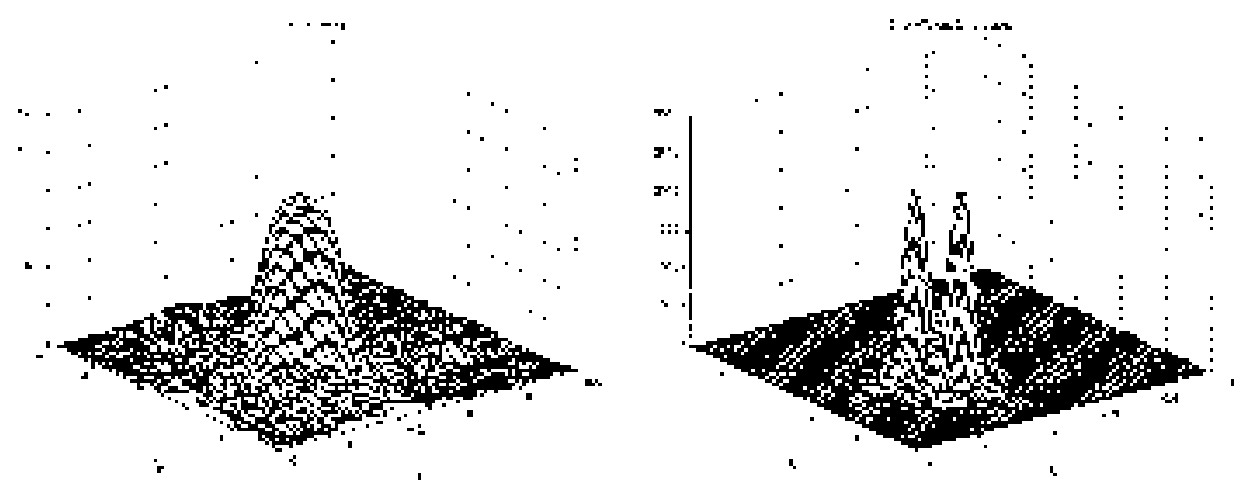

Figure 3. a) Fourier image (rectangular window), b) 2-D AWNE image (high resolution).

\subsection{Implementation Issues and Simulated Point Scatterer Examples}

The AWNE procedure involves the use of a window which can be chosen by the user. Throughout most of this paper, we will restrict our choice of window type and length to simplify our comparisons. Given the length $L \times L$ set of data ( $L$ odd) to extrapolate, a circularly symmetric [23] Hamming window of diameter $J=L$ is used in most cases in the application of the 2-D AWNE. A window length $J=L$ is used for the 1-D AWNE and for the Taylor window (1-D or 2-D separable) that is used when the Fourier imaging procedure is applied. This window length that matches the data size is a conservative choice that incorporates only original data samples and thus produces a convergence at iteration 2 due to the operation in Step 3 and the form of $p(n, m)$ defined in Step 0. It also imposes a maximum extrapolation length of $N_{E} \times N_{E}$, where $N_{E}$ is approximately three times the given data sample length $L$, see Eq.(3). Figure $3 a$ ) shows the spectrum of a square of $17 \times 17$ samples of the $2-\mathrm{D}$ signal given by

$$
x(n, m)=e^{j 2 \pi(0.2 n+0.16 m)}+e^{j 2 \pi(0.15 n+0.2 m)}
$$

using a rectangular window where it is clear that the sinusoids cannot be resolved. Figure $3 \mathrm{~b}$ ) shows the spectrum of the $51 \times 51$ samples that result after the application of the 2-D AWNE to the given data. The high resolution characteristics of the resulting spectrum can be observed.

In order to contrast the performance of the 1-D and 2-D versions of AWNE with the Fourier method, Figure 4 shows the results of the three methods on a collection of simulated point scatterers configured to spell $\Sigma E R I M$ used by DeGraaf in [1]. The data is corrupted by additive white Gaussian noise with a target-to-noise ratio of $33 \mathrm{~dB}$. Figure 4 shows a) the true points; b) the Fourier image of the $24 \times 24$ samples of original phase history with rectangular windowing; c) the Taylor windowed Fourier image; d) the spectrum of the $72 \times 72$ samples of extrapolated phase history computed 

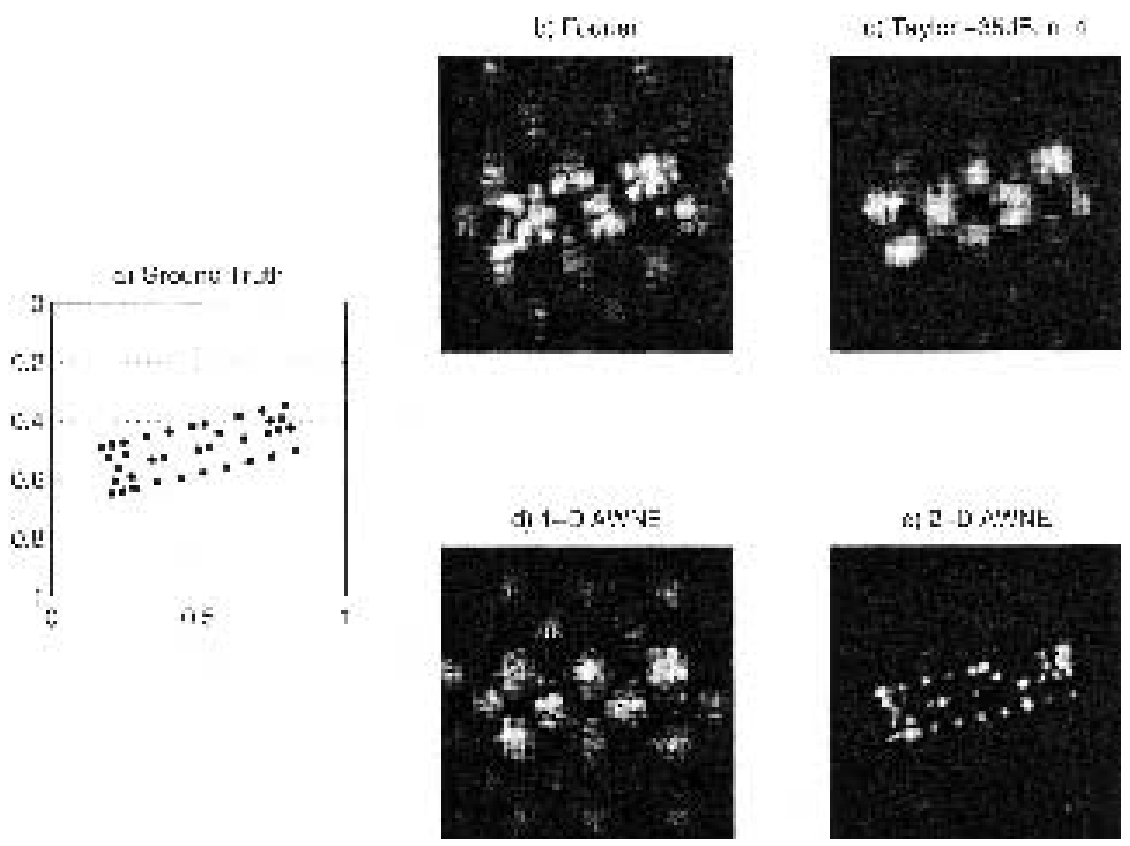

Figure 4. Simulated point scatterer Images.

with the 1-D AWNE method in a row-column separable fashion; and e) the spectrum of the $72 \times 72$ phase history computed with the 2-D AWNE method. Note that all the magnitude images presented in this paper are plotted on a square root scale. It is interesting to see the good localization of the point scatterers produced by the 2-D AWNE, the absence of sidelobes in the image, and the improvement over the results produced by its 1 -D counterpart.

\section{Processing MSTAR SAR Images with 2-D AWNE}

One of the reasons for implementing and presenting the 2-D AWNE method in this paper is to improve our previous results obtained when the 1-D version [7] was applied to actual SAR imagery. The images selected from the MSTAR public target database [12] are formed using the Sandia method [24] which uses an overlapped-subaperture algorithm based on FFT and vectorial operations to allow for real-time and fine resolution operation. Among the tasks that the algorithm performs are motion compensation, phase correction, Taylor windowing and DFT computation. This database includes SAR images of different time-critical targets at different depression and aspect angles. The two images used for experimentation throughout this paper include a T72 tank. 


\subsection{Obtaining the Phase History}

To produce a new image, the AWNE method works on the phase history, which is obtained by inverting the last two steps of the MSTAR image formation process. The size of an MSTAR target image is $128 \times 128$ complex pixels. After performing the DFT, the phase history revealed is approximately $100 \times 100$ in dimension. From the header file of the MSTAR image, we know that a $-35 \mathrm{~dB}$ Taylor window is used and we assume that $\bar{n}=4$ is used. Thus, we form the corresponding Taylor window and divide it out from the $100 \times 100$ phase history samples to obtain the unweighted frequency (phase history) samples. These phase history samples are ready for the application of the 1-D AWNE [7] or the 2-D AWNE algorithms. It is important to mention that we take advantage of all the previous processing done by the Sandia image formation method to focus our attention exclusively on the effects of the extrapolation procedure.

\subsection{Applying the 2-D AWNE}

The application of the 2-D AWNE to the MSTAR images is intended to show the improvement in some of the characteristics of the SAR imagery when this superresolution method is used in lieu of the traditional Fourier method. There are several experiments defined to evaluate the performance of the AWNE algorithm. The first experiment is done to evaluate the two versions of AWNE. In the previous section, we have shown that the 2-D version gives better results than the 1-D version (see Figure 4) when the methods were applied to simulated point scatterers. The application of both algorithms is presented in Figure 5 to contrast their performance with the Fourier (MSTAR) method as a basis for comparison under a handicapped situation. For fairness purpose, extrapolations that go beyond the original data size are truncated to this size. Figure 5 shows in a) the original MSTAR image (hb03793.015); in b) the image formed after the extrapolation of the middle $49 \times 49$ phase history samples to $100 \times 100$ samples using 1 -D AWNE $(1$ iteration $)$ in a row/column fashion; and in c) the image formed by the application of the 2-D AWNE

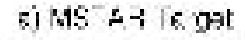

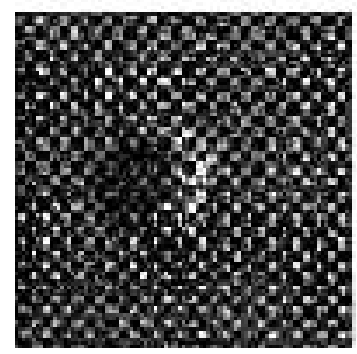

c) $1-0$ sistif $43 \times 49$

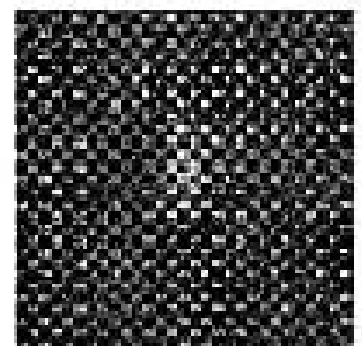

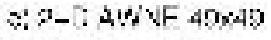

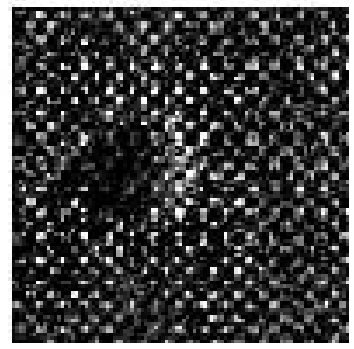

Figure 5. a) Original MSTAR image, b) 1-D and c) 2-D handicapped SR images, obtained by extrapolating a subset of $49 \times 49$ phase history samples to $100 \times 100$. 
( 1 iteration) to extrapolate from $49 \times 49$ to $100 \times 100$ phase history samples. Two important things can be observed by evaluating the 2-D AWNE result of Figure $5 \mathrm{c}$ ): the preservation of the target's shadow present in Figure 5a) and the absence of the central sidelobes present in Figure 5b). The image in Figure 5c) is closer to the MSTAR image in Figure 5a) than its counterpart in Figure 5b). The scatterers are well defined even though the image has been generated with only a quarter of the phase history samples that produced the original MSTAR image. Some objective metrics were presented in [7] and [8] to evaluate quantitatively the performance of both SR versions of the AWNE method in this handicapped way using the original MSTAR image as a basis for comparison.

There is an inherent drawback to the 2-D AWNE method imposed by the need to solve $L^{2}$ linear equations per iteration of the algorithm. Motivated by the decimation/mosaicing strategy employed by DeGraaf [1], we propose two schemes for the application of the 2-D AWNE version: a zooming on the targets or region of interest to reduce the amount of data, and similarly a partitioning of the image in the spatial domain that renders a small set of phase history to extrapolate for each block. For the zooming scheme, before applying the 2-D AWNE method, the phase history is modulated to baseband by image-domain shifting, lowpass filtered, and then decimated by 3 in both range and cross-range directions. These operations result in a zooming of the target part of the MSTAR SAR image (see Figure 6). Then the decimated phase history can be used to perform full rather than handicapped extrapolation experiments (i.e. extrapolate the available data to a larger size than the given original phase history size in order to produce a higher resolution image). For the partition scheme, we break the complex image into blocks and recover the phase history of each one in an independent way. Then, the 2-D AWNE is applied to every block of phase history to obtain an extrapolated block of phase history and then a new image block that can be reassembled with the other blocks as shown in the block diagram in Figure 7. The following section will discuss the results from these experiments in further detail.

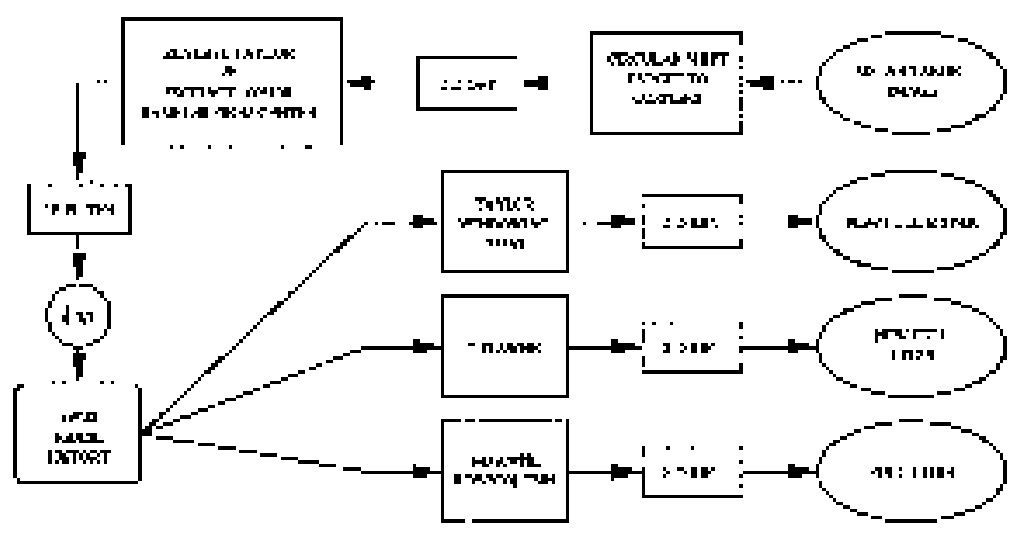

Figure 6. Block diagram for the zooming MSTAR experiments. 


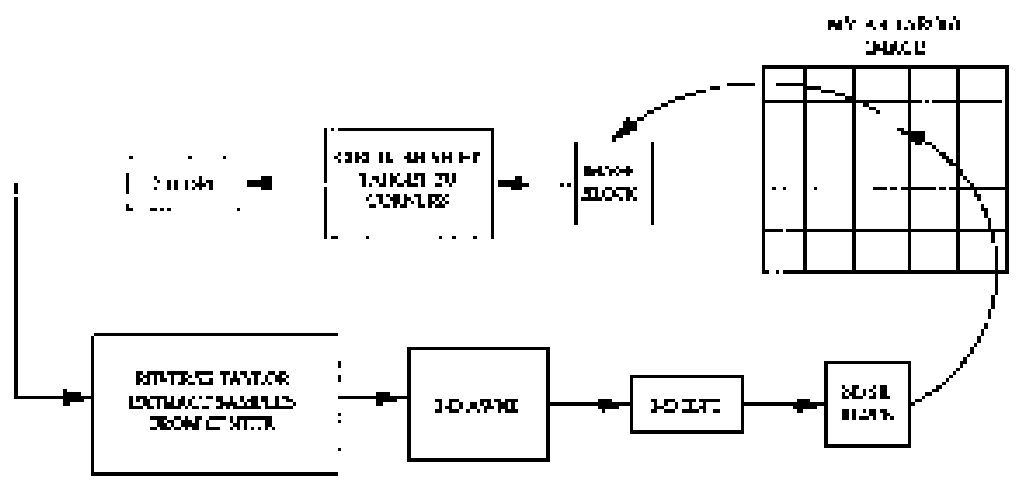

Figure 7. Block diagram for the partitioning MSTAR experiments.

\section{Experimental Comparison of Fourier vs. Superresolved Images}

We present some experiments to evaluate the quality of the superresolved (SR) images in comparison to Fourier images. Figure 6 shows a block diagram which summarizes the set of experiments carried out on the target chip or Region Of Interest (ROI) which covers one-ninth of the original image area. Using the zooming procedure defined in the previous section, we obtain the new set of $33 \times 33$ Phase History $(\mathrm{PH})$ samples of the target ROI. Using this new phase history, we form the new full MSTAR (Fourier) image which is used as the reference or ground truth in the comparison done for the evaluation of the SR images.

\subsection{Target Zooming Scheme}

The full resolution experiments explore the possibility of going beyond the resolution limits implied by the given phase history data size. An example of extrapolated phase history samples obtained with the 2-D AWNE method applied to the set of $33 \times 33$ samples to extrapolate to $100 \times 100$ samples using only one iteration (a conservative choice) corresponding to the target chip can be seen in Figure 8a). Recall that the original $33 \times 33$ block of phase history data is preserved perfectly (centered at the 50th location) in the middle of the extrapolated set. Note that the AWNE method gives a natural amplitude decay away from the given data region which prevents sidelobe interference in the resulting image. Figure 9 shows: a) the new full MSTAR Fourier image (hb03793.015); b) the full 1-D (row/column separable) SR image; and c) the full 2-D SR image. Higher resolution in the point scatterers can be observed in Figures 9 b) and c) which can be better appreciated in the 3-D mesh plots presented in Figures 10 a) and b). The comparison of these with the 3-D plot of the new full MSTAR presented in Figure 8b) clearly favors the SR images in terms of resolution although the extrapolation lengths are minimal (a 3 fold increase). 

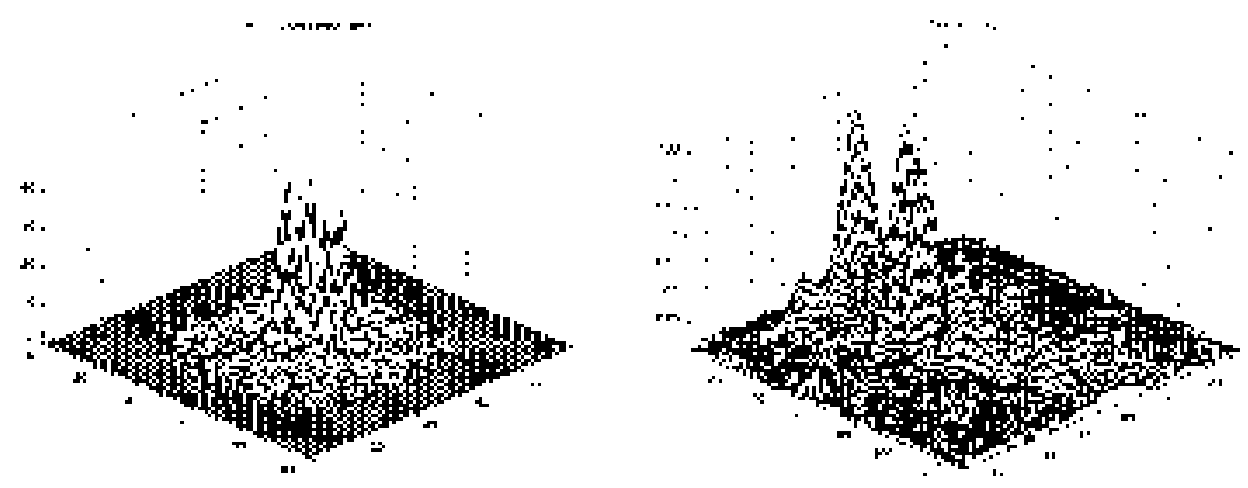

Figure 8 . a) Magnitude of the extrapolated phase history from $33 \times 33$ to $99 \times 99$, b) New Full MSTAR 3-D plot.

Further analysis of the effects of extrapolation using the 2-D AWNE method can be done on Figure 11 where structural information is presented in terms of the contour plots and the convex hulls of the prominent point scatterers for each case. Figure 11 shows: a) the full new MSTAR image; and b) the full 2-D SR image. In Figure 11, the convex hull of the prominent point scatterers represents the constellation of the target. This is a geometric representation that can be used in peak-based target detection [25] by comparing as a means of constellations of peaks. The general shape of the constellation of the peaks of Figure 11a) for the new full MSTAR image is preserved in the 2-D SR image (Figure 11b)), where a better definition of this constellation can be observed. Furthermore, the contour plots in the same Figure show how the resolution of the point scatterers is superior in the full 2-D SR image and how the process also suppresses clutter around the target. From a subjective point of view, the conclusion that going beyond the original limit of phase history samples produces better images is easily established. However, objective comparison metrics cannot be used with these full SR images, as in previous work

E.) toullar Imaga

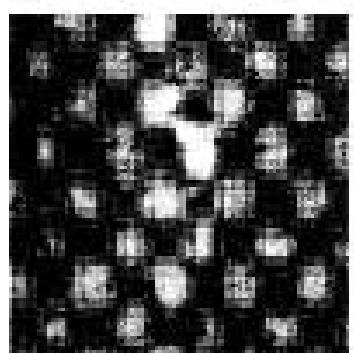

b: 1-v.s?rve

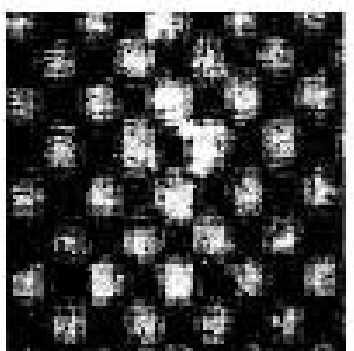

9i

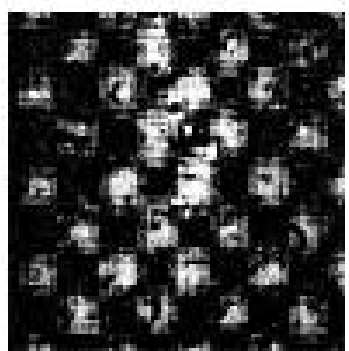

Figure 9. a) New Full MSTAR, b) Full 1-D and c) Full 2-D SR images. 

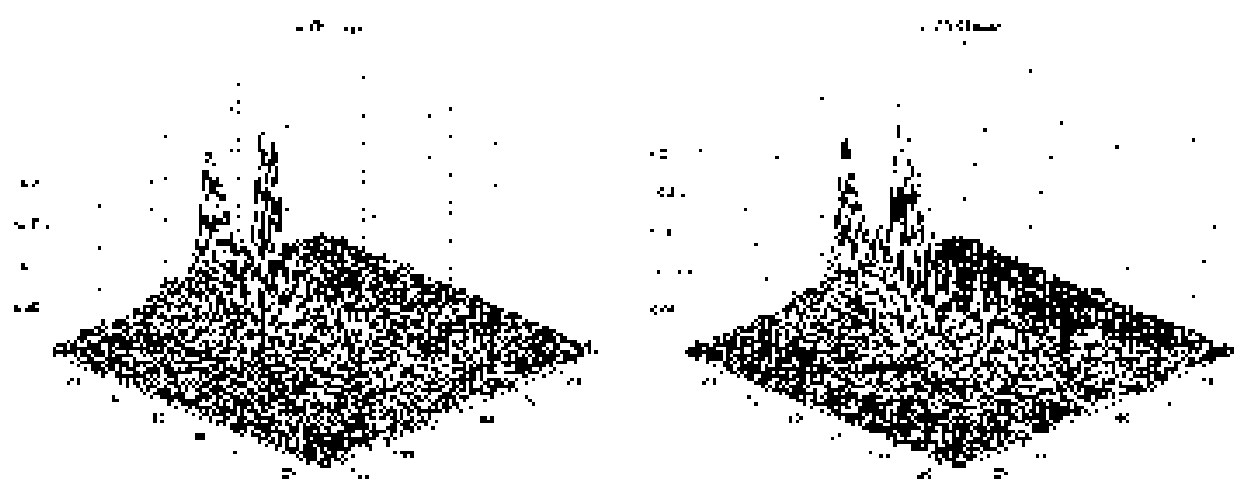

Figure 10. a) Full 1-D SR 3-D plot, b) Full 2-D SR 3-D plot.

reported in [7], [8] because of the lack of a ground truth image to compare with. An alternative approach is the objective evaluation of the full SR images based on their impact on the performance of an ATR system [2], [26]. Also, general image quality metrics that allow a comparison among various superresolution generated images coming from different methods [1], [3], [4], [5], [6] can be defined and used.

\subsection{Block Partition Scheme}

Here we present the results of the scheme to save computational effort described by the block diagram in Figure 7. It is an approach to reduce the amount of data to extrapolate at any given time while still processing the complete phase history data available. Figures 12
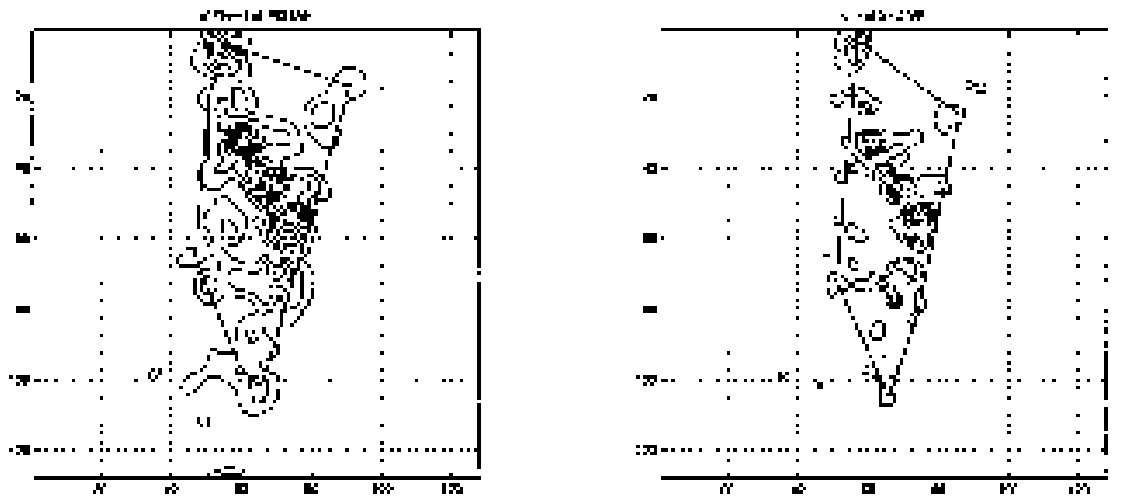

Figure 11. a) New Full MSTAR, and b) Full 2-D SR contour and convex hull plots. 


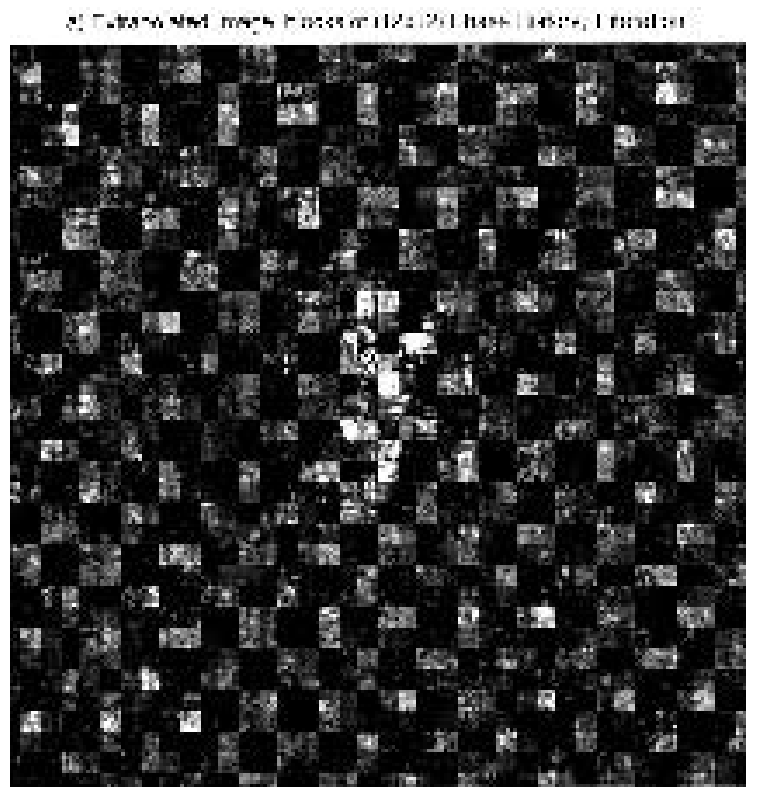

Figure 12. Extrapolated image using block partitioning with extrapolation length of 3 times the original block size.

and 13 show two images processed in a partition scheme of blocks of size 16 by 16 pixels each from which we obtain $12 \times 12$ phase history samples. The images are processed with the 2D AWNE using parameters that lead to extrapolated blocks that are 3 times and 5 times larger. Both images show the preservation of the scatterer centers and the attenuation of clutter. In addition, both images present the shadow of the target as seen before in Figure 5c) and in the original MSTAR image, see Figure 5a). However, we can appreciate higher resolution in the images of Figure 12 and Figure 13 than in the ones of Figure 5 because the former ones make use of the full set of phase history data while the latter ones use only a subset of the data in a handicapped situation. An interesting behavior in the performance of our algorithm is the sparse result that is produced when large extrapolation ratios and several iterations are used. The image in Figure 13 formed with 5 fold extrapolation has better concentration of the scatterers and shows more background clutter suppression. Further experimental results with larger extrapolation lengths showed a very sparse image with single peaks representing the scatterer centers that can be used as features for an ATR system.

Clearly, the independent processing of the superresolved blocks introduces blocking artifacts as it can be observed in Figures 12 and 13. The artifacts are better appreciated in Figure 12. These artifacts are expected to affect CFAR detection applied to the image by skewing the image pixel distribution and generating false-positives outside the target region. Approaches to deal with the artifacts can be postprocessing at the boundaries, or block overlapping and averaging as in [1]. 


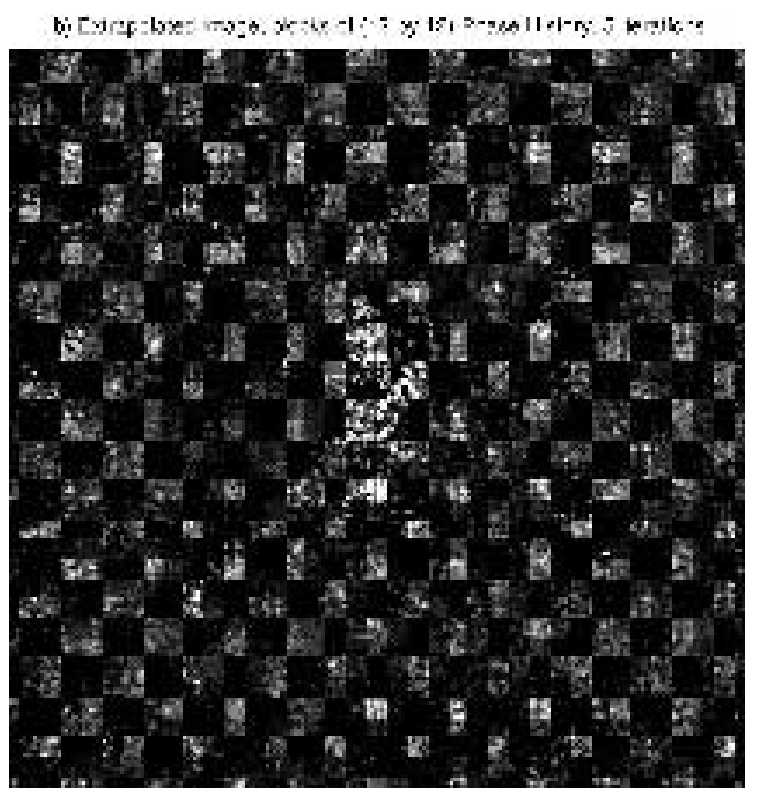

Figure 13. Extrapolated image using block partitioning with extrapolation length of 5 times the original block size.

\section{Experimental Comparison to Other Superresolution Methods}

In this section, Capons' minimum variance method (MVM) and the 2-D linear prediction or AR method are used to form superresolved SAR images. They are compared visually with the 2-D AWNE images under handicapped situations using a different MSTAR image than before. The main idea is to contrast particular features of the superresolution SAR formation methods. The superresolution methods process a subset of the full phase history (PH) samples to form SR SAR images of equivalent spectral resolution and size. As noted before, the handicapped situation can be used to stress the benefits of using superresolution methods when only limited data is available.

The 2-D linear prediction or autoregressive (AR) method [27], [28] is implemented using a quarter-plane support modified covariance version. In particular, the method uses a fast Cholesky-decomposition type of algorithm [29] and the 2-D AR power spectral density is produced by the combination of the results for quadrants 1 and 4 [28]. For this experiment the PH signal subset used is $55 \times 55$ and the order of the 2-D AR parameter array chosen is $22 \times 22$. Figure 14 illustrates the $2-\mathrm{D}$ AR SR image which presents an unusual character for the target although a few prominent point scatterers are well defined. In addition, it can be observed that the shadow of the target is not well preserved. Furthermore, the introduction of spurious point scatterers throughout the background region can be observed. These are undesirable image characteristics for ATR systems based on prominent peak processing [26]. 


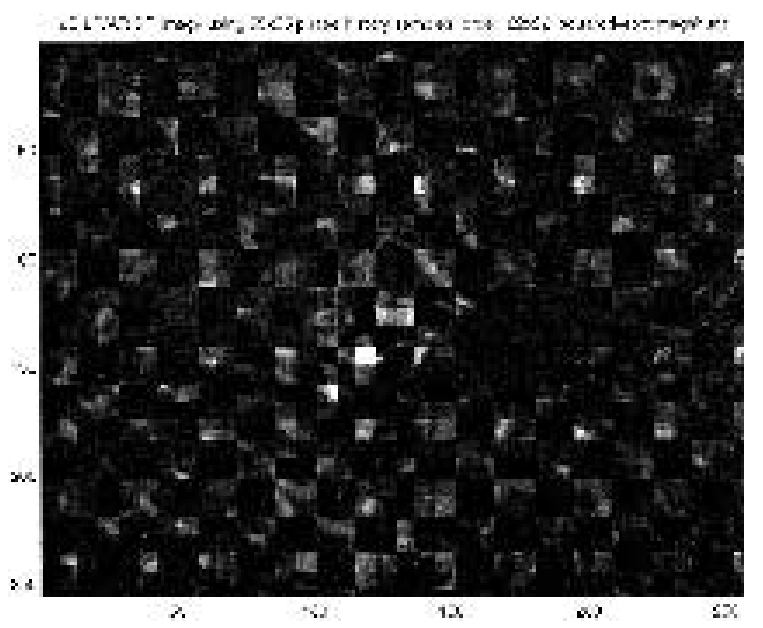

Figure 14. 2-D (AR) linear prediction SR image (hb03787.015) using $55 \times 55$ phase history samples and a filter order of $22 \times 22$.

The 2-D version of Capon's MVM [27], [28] is a popular choice in SAR imaging [1], [2], [3], [29], [30] and recently there has been increasing interest in its computational aspects. The interest comes from the argument that the main burden of the MVM does not come from the correlation estimate formation and inversion but from the computation of the estimator over all the needed frequencies. Therefore, fast algorithms to overcome this problem have been proposed in [29], [30]. For the calculation of the spectral estimate, the forwardbackward averaged correlation matrix is formed using all possible $M_{1} \times M_{2}$ subapertures, which are available from the selected PH sample subset that forms a matrix of size $N_{1} \times N_{2}$, leading to a number of subapertures given by $A=\left(N_{1}-M_{1}+1\right)\left(N_{2}-M_{2}+1\right)$. Empirical results [1] suggest that the MVM produces satisfactory results when the relation between subaperture size and aperture size is kept between the following bounds

$$
0.4 \leq \sqrt{\frac{M_{1} M_{2}}{N_{1} N_{2}}} \leq 0.5
$$

For the computation of the experimental results shown next, a direct implementation of the MVM is used and the empirical bound in Eq.(7) is satisfied. The next set of figures show the results for the case when approximately a quarter $(55 \times 55)$ of the full phase history data set is used to generate the superresolved images using a $256 \times 2562$-D DFT. Figure 15 and Figure 16 show the corresponding superresolved images for the 2-D AWNE and Capon methods. In this case, the SR MVM image has better definition of the main scatterer center but the smoothness presented in the rest of the target area may be an undesirable feature. In addition, the shadow for the SR MVM image is almost perfectly preserved. On the other hand, the SR 2-D AWNE image has a better definition of the prominent peaks, is comparable in terms of the shadow preservation, but is inferior as far 


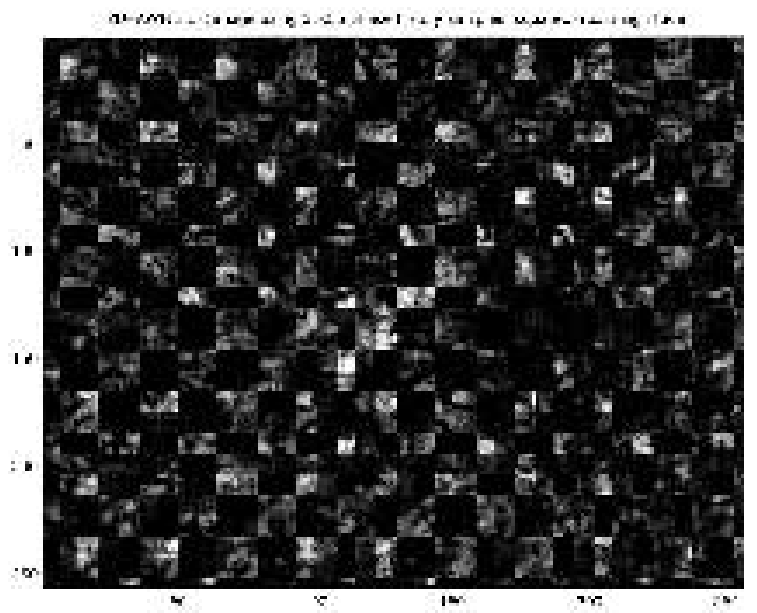

Figure 15. 2-D AWNE SR image (hb03787.015) extrapolating $55 \times 55$ to $163 \times 163$ phase history samples.

as speckle reduction and clutter suppression. Overall, the SR images are superior in comparison with the Fourier images (not shown) using the same subset of phase history samples.

A hybrid scheme between the 2-D AWNE and the MVM is proposed to produce a SR image with the best features of both methods. The main goal is to achieve the high resolution of the prominent scatterer centers produced by the 2-D AWNE and the better

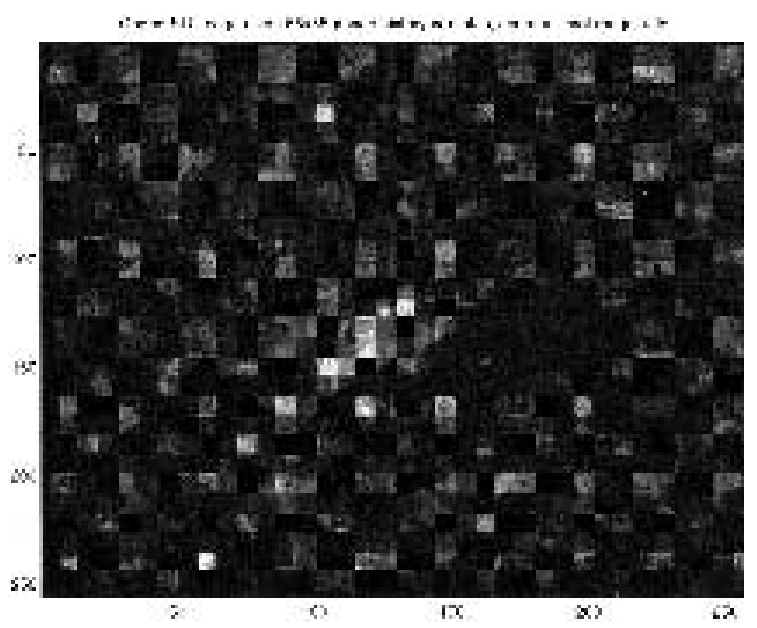

Figure 16. Capon SR image (hb03787.015) using $55 \times 55$ phase history samples. 


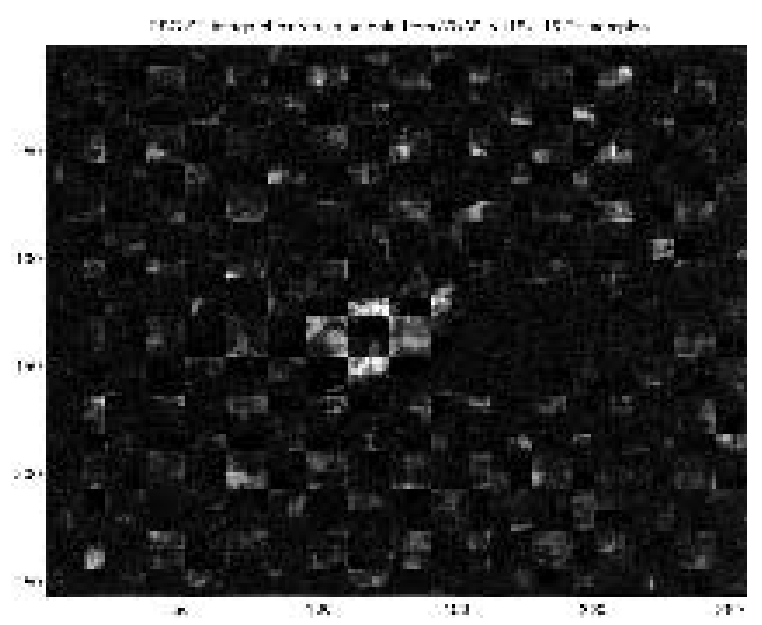

Figure 17. Hybrid Scheme. Capon via AWNE SR image (hb03787.015) using $39 \times 39$ phase history samples.

clutter suppression and speckle reduction produced by the MVM. To illustrate the viability of this scheme a $39 \times 39$ subset of phase history samples is extrapolated using the $2-\mathrm{D}$ AWNE to $115 \times 115$ and then these samples are used by the MVM to produce a SR image. In the resultant SR image shown in Figure 17, it can be observed that the scatterer centers have better resolution than in the MVM SR image (Figure 16) and the clutter suppression and speckle reduction is better than in the 2-D AWNE SR image (Figure 15) even when the subset of original PH samples used is about $50 \%$ of the $55 \times 55$ set. This leads us to believe in the potential of complementing the hybrid scheme to produce superior SR SAR imagery than either method alone at expense of higher complexity.

\section{Conclusions}

We have presented a detailed two-dimensional (2-D) extension of the adaptive weighted norm extrapolation method and proposed its application to achieve superresolution in SAR imaging by showing its benefits on simulated and MSTAR images. The use of the 2-D AWNE procedure instead of the 1-D version [7] results in a better image with less undesirable artifacts. The 2-D AWNE proved to have better performance in the handicapped experiments realized. First, it preserved the shadow of the target and produced a closer image to the MSTAR reference (see Figure 5). The other focus of our experiments was the illustration of the potential advantages of going beyond the traditional limits of resolution by extrapolating the full aperture of phase history to a larger size. We proposed two schemes for the formation of images that allow the full use of phase history data to produce an extrapolation using the 2-D AWNE algorithm. We analyzed performance by visual comparison and by comparison of the geometric constellation of prominent point 
scatterers of the targets present in the images. The 2-D AWNE method proved to be superior to its 1-D counterpart and it was established that going beyond the original limit of phase history samples produces better images (see Figure 12 and 13).

\section{Acknowledgments}

The authors would like to thank Dr. Stuart DeGraaf and Dr. Armin Doerry for suggestions and valuable discussions. We also thank Dr. DeGraaf for providing the data for the simulated point scatterers of Figure 4 and S. Lawrence Marple, Jr. for providing the code for the 2-D (AR) linear prediction method.

\section{References}

1. S.R. DeGraaf, "SAR Imaging via Modern 2-D Spectral Estimation Methods," IEEE Trans. Image Process., vol. 7, May 1998, pp. 729-761.

2. L.M. Novak, G.R. Benitz, G.J. Owirka, and L.A. Bessette, “ATR Performance Using Enhanced Resolution SAR,” in SPIE, vol. 2757, Orlando, FL, April 1996, pp. 332-337.

3. J. Li and P. Stoica, “An Adaptive Filtering Approach to Spectral Estimation and SAR Imaging," IEEE Trans. Signal Process., vol. 44, June 1996, pp. 1469-1484.

4. Z. Bi, J. Li, and Z.S. Liu, "Super Resolution SAR Image Formation via Parametric Spectral Estimation Methods," in SPIE, vol. 3370, Orlando, FL, April 1998, pp. 238-249.

5. J. Li, P. Stoica, Z. Bi, R. Wu, and E.G. Zelnio, "A Robust Hybrid Spectral Estimation Algorithm for SAR Imaging," in 32nd Asilomar Conf. on Signal, Systems and Computer, vol. 1, Pacific Grove, CA, October 1998, pp. $1322-1326$.

6. F.M. Candocia and J.C. Principe, "A Method Using Multiple Models to Superresolve SAR Imagery," in SPIE, vol. 3370, Orlando, FL, April 1998, pp. 197-207.

7. A.E. Brito, S.H. Chan, and S.D. Cabrera, “Application of AWNE Superresolution to MSTAR SAR Images," in Proc. of IASTED SIP'98, vol. 1, Las Vegas, NV, May 1998, pp. 495-499.

8. A.E. Brito, S.H. Chan, and S.D. Cabrera, "SAR Image Formation Using 2-D Re-Weighted Minimum Norm Extrapolation," in SPIE Algorithm for SAR Radar Imagery VI, vol. 3721, Orlando, FL, April 1999, pp. 78-91.

9. S.D. Cabrera and T.W. Parks, "Extrapolation and Spectral Estimation with Iterative Weighted Norm Modification," IEEE Trans. Signal Process., vol. 39, April 1991, pp. 842-851.

10. M.D. Sacchi, T.J. Ulrych, and C.J. Walker, "Interpolation and Extrapolation Using a High-Resolution Discrete Fourier Transform," IEEE Trans. Signal Process., vol. 46, January 1998, pp. 31-38.

11. A.E. Brito, "Iterative Adaptive Extrapolation Applied to SAR Image Formation and Sinusoidal Recovery," $\mathrm{PhD}$ thesis, Department of Electrical and Computer Engineering, The University of Texas at El Paso, El Paso, TX, 2001.

12. "Sensor Data Management System Page," http://www.mbvlab.wpafb.af.mil/public/MBVDATA.

13. A.K. Jain and S. Ranganath, "Extrapolation Algorithms for Discrete Signals with Application in Spectral Estimation," IEEE Trans. Acoust. Speech Signal Process, vol. ASSP-29, September 1981, pp. 830-845.

14. A. Papoulis, "A New Algorithm in Spectral Analysis and Band-Limited Extrapolation," IEEE Trans. Circuits Syst., vol. CAS-22, September 1975, pp. 735-742.

15. C.L. Byrne and R.M. Fitzgerald, "Reconstruction from Partial Information with Applications to Tomography," SIAM Journal on Applied Mathematics, vol. 42, August 1982, pp. 933-940. 
16. C.L. Byrne and R.M. Fitzgerald, "Spectral Estimators that Extend the Maximum Entropy and Maximum Likelihood Method," SIAM Journal on Applied Mathematics, vol. 44, April 1984, pp. 425-442.

17. L. Potter and K. Arun, "Energy Concentration in Band-Limited Extrapolation," IEEE Trans. Acoust. Speech Signal Process., vol. 37, July 1989, pp. 1027-1041.

18. E. Diethorn and J. Munson, D.C., "A Linear, Time-Varying System Framework for Noniterative DiscreteTime Band-Limited Signal Extrapolation,” IEEE Trans. Signal Process., vol. 39, January 1991, pp. 55-68.

19. S. Dharanipragada and K. Arun, "Bandlimited Extrapolation Using Time-Bandwidth Dimension," IEEE Trans. Signal Process, vol. 45, December 1997, pp. 2951-2966.

20. S.H. Chan, A.E. Brito, and S.D. Cabrera, "Characterization of Windowing Effects in Adaptive Extrapolation of Sinusoids," in 33rd Asilomar Conf. on Signals, Systems and Computers, vol. 2, Pacific Grove, CA, October 1999, pp. 1092-1097.

21. S.H. Chan, A.E. Brito, and S.D. Cabrera, "Imposed Window Estimate for Adaptive Extrapolation of Complex Sinusoids in Gaussian Noise," in 9th IEEE DSP Workshop, Hunt, TX, October 2000.

22. A.E. Brito and S.D. Cabrera, "Analysis of Autocorrelation Pursuit via Non-Parametric Iterative Extrapolation," in 35th Annual Conference on Information Sciences and Systems, vol. 2, Baltimore, MD: Johns Hopkins University, March 2001, pp. 633-638.

23. J.S. Lim, Two-Dimensional Signal and Image Processing, Englewood Cliffs, New Jersey: Prentice-Hall, 1990 .

24. B.L. Burns and J.T. Cordaro, "A SAR Image-Formation Algorithm that Compensates for the SpatiallyVariant Effects of Antenna Motion,” in SPIE, vol. 2230, Orlando, FL, April 1994, pp. 14-24.

25. W.W. Irving, R.B. Washburn, and W.E.L. Grimson, "Bounding Performance of Peak-Based Target Detectors," in SPIE, vol. 3070, Orlando, FL, April 1997, pp. 245-257.

26. B. Bhanu and G. Jones III, "Performance Characterization of a Model-Based SAR Target Recognition System Using Invariants," in SPIE, vol. 3070, Orlando, FL, April 1997, pp. 305-321.

27. S.M. Kay, Modern Spectral Estimation, Englewood Cliffs, NJ: Prentice Hall, 1988.

28. S.L. Marple, Digital Spectral Analysis with Applications, Englewood Cliffs, NJ: Prentice Hall, 1987.

29. A. Jakobsson, S.L. Marple, and P. Stoica, "Computationally Efficient Two-Dimensional Capon Spectrum Analysis,” IEEE Trans. Signal Process., vol. 48, September 2000, pp. 2651-2661.

30. E.G. Larsson and P. Stoica, "Fast Implementation of Two-Dimensional APES and Capon Spectral Estimators," in Proc. IEEE ICASSP, vol. V, Salt Lake City, UT, March 2001, pp. 3069-3072. 